\title{
Du stéthoscope et de ses évolutions en tant que symbole
}

\section{Eberhard Wolff}

Prof. Dr rer. soc., rédacteur culture, histoire, société

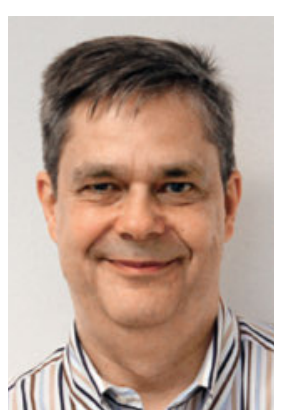

J'ai récemment lu dans un journal un article sur le test «MoCa», à l'aide duquel le médecin personnel de Donald Trump a évalué ses facultés cognitives. Une photo présentait l'auteur du test, le neurologue libano-canadien Ziad Nasreddine. On l'y voit en chemise et cravate, devant un écran d'ordinateur, apparemment en train de fournir énergiquement des explications. Un stéthoscope pend négligemment autour de son cou. ${ }^{1}$

Il semblerait que Monsieur Nasreddine pratique effectivement la médecine en tant que spécialiste de la maladie d'Alzheimer. Il n'empêche que j'ai un peu de mal à imaginer qu'il ait écouté la cage thoracique, le cœur ou les intestins de l'un de ses patients neurologiques à l'aide du stéthoscope juste avant la séance photo et qu'il n'ait pas eu le temps de l'enlever. Une autre photo disponible sur le Web le montre devant un mur couvert de diplômes encadrés, cette fois en costume complet, et toujours avec un stéthoscope autour du cou. Je ne veux pas le froisser, mais l'époque où le médecin examinait ses patients en veston ou veste de costume est révolue depuis plusieurs générations. S’ils ne portent plus la blouse blanche, les médecins travaillent aujourd'hui en chemise. Je soupçonne que le stéthoscope est ici davantage employé comme un symbole que comme un instrument manuel. Il a cela de commun avec les diplômes. Les deux ont une fonction pratique, mais ils communiquent aussi des messages.

Bon, peut-être que ce sont les différents photographes qui ont dit: «Monsieur Nasreddine, passez-vous donc un stéthoscope autour du cou, pour qu'on sache que vous êtes médecin en dépit du fait que vous ne portez pas la blouse blanche.» Nous l'ignorons. Mais les stéréotypes sont souvent créés par les personnes extérieures.

Ce que nous savons, en revanche, c'est qu'au cours du siècle dernier, le stéthoscope, dans sa forme moderne, a évolué en parallèle de la blouse blanche en tant que signe distinctif de la profession de médecin et que symbole de la compétence et de l'autorité médicales. Il y a longtemps, j'ai lu dans un quotidien américain l'interview d'un médecin qui intervenait en tant qu'expert chevronné auprès des tribunaux. Il expliquait que cela faisait des années qu'il n'avait plus pratiqué, mais qu'il avait toujours dans sa mallette un stéthoscope qu'il sortait lorsque le besoin s'en faisait sentir. Celui-ci lui conférait davantage d'autorité médicale.

La mesure classique de la tension à l'aide du stéthoscope a contribué à l'essor de ce symbole d'autorité. Lorsqu'elle s'est généralisée dans les cliniques aux alentours de 1900, beaucoup de médecins exigeaient d'être seuls autorisés à utiliser cette innovation. Mais au milieu du siècle dernier, la tension était devenue un examen de routine, de plus en plus souvent effectué par du personnel non médical. Un marché s'est alors développé pour ce dernier: aux États-Unis dans les années 60, par exemple, des stéthoscopes se distinguant par leur nom («assistoscope», «nurse-o-scope»), leurs tons pastel et leur matière légère des lourds stéthoscopes noirs métalliques des médecins étaient disponibles dans le commerce. ${ }^{2}$ Et pourtant, je pense que c'est au plus tard à ce moment-là que le déclin de ce symbole d'autorité est devenu inéluctable.

L'une de mes caricatures médicales préférées ${ }^{3}$ montre un patient assis sur une table d'examen. Le médecin se tient à côté de lui en blouse blanche, avec stéthoscope et miroir frontal. Le patient lui demande: «Au fait, à quoi sert ce truc sur votre tête?» Le médecin répond: «À nous identifier en tant que médecins dans les B. D.» Le symbole d'autorité devient un cliché. Et on notera qu'il s'agit ici du miroir frontal, aujourd'hui largement remplacé par des appareils d'examen à piles. Le stéthoscope, lui, pend presque invisible autour du cou.

Une analyse impressionniste d'images sur Internet montre que le stéthoscope est aujourd'hui toujours associé au médecin - homme ou femme. Mais il symbolise aussi la médecine en général. Les costumes de carnaval sont une merveilleuse source de clichés (fréquemment désuets). Là, le stéthoscope, associé à la coiffe, fait souvent partie de la tenue d'infirmière.

Il symbolise aussi, de manière très générale, le diagnostic. C'est par exemple l'icône de l'application «Utilitaire de disque» sur les ordinateurs Mac. Le stéthoscope en tant que signe de l'autorité médicale n'est donc plus ce qu'il était. Je mets en garde Monsieur Nasreddine et tous ses collègues: se faire prendre en photo avec un stéthoscope n'est pas forcément une bonne idée. Un de ces jours, on risque de leur demander d'examiner de plus près un disque dur. 